\title{
Bei über 30-Jährigen versagt die Therapie häufiger
}

\author{
Bei Asthmatikern über 30 Jahre ist es im Vergleich zu jüngeren Patienten viel wahrscheinlicher, \\ dass die Therapie versagt. Das geht aus einer Analyse von zehn Studien hervor.
}

Daten etwa der „Centers for Disease Control and Prevention“" (CDC) legen nahe, dass mit dem Altern sowohl Morbidität als auch Mortalität bei Asthmapatienten zunehmen. Doch bisher gab es keine Untersuchung, in der explizit versucht wurde, den Stellenwert von Alter und Geschlecht für den Erfolg einer Asthmatherapie zu überprüfen. Deshalb haben Pneumologen und Asthmaforscher um Ryan M. Dunn u. a. von der Universität von Colorado in Denver die Befunde von 1200 Patienten (680 Frauen) ausgewertet, die an 10 Studien des „Asthma Clinical Research Network" (ACRN) zwischen 1993 und 2013 teilgenommen hatten (Am J Respir Crit Care Med 2015: online 11. Juni).

Bei allen Patienten, die vor allem an größeren Kliniken und in Facharztpraxen versorgt wurden, war ein leichtes bis mittelschweres Asthma diagnostiziert worden. Patienten mit Exazerbationen innerhalb des Monats vor Aufnahme von Teilnehmern in die jeweilige Studie sowie Raucher waren ausgeschlossen. 579 Patienten waren mindestens 30 Jahre alt, 303 über 37 Jahre. Im Fokus der statistischen Analyse stand der Anteil der Patienten mit Therapieversagen in den jeweiligen Untersuchungsgruppen.

Als Versagen der Therapie galt u. a. eine Asthmaexazerbation mit Bedarf an oralen Kortikosteroiden oder die Notwendigkeit einer Notfallversorgung, eine Verschlechterung der Lungenfunktion oder ein erhöhter Medikamentenbedarf. Wie Dunn und Kollegen berichten, kam es im Alter von mindestens 30 Jahren bei signifikant mehr Patienten zu einem Therapieversagen als bei jüngeren (17,3 vs.10,3\% bzw. 64/621 vs. 100/579 Patienten). Die Wahrscheinlichkeit dafür war um 82 Prozent erhöht.

\section{COPD erhöht Infarktrisiko, aber nicht das Schlaganfallrisiko}

COPD-Patienten leiden häufig an Komorbiditäten und gerade Herz-Kreislauf-Erkrankungen limitieren die Lebenserwartung. In einer sehr großen Studie bei Patienten mit Vorhofflimmern wurde nun ermittelt, dass die COPD per se die Risiken für Schlaganfall oder systemische Embolien nicht erhöht, wohl aber die Risiken für KHK, Herzinfarkt und Herzinsuffizienz.

An der ARISTOTLE-Studie, die Apixaban mit Warfarin zur Schlaganfallprophylaxe bei Vorhofflimmern verglich, hatten 18.134 Patienten teilgenommen, darunter 1950 Patienten mit COPD. Diese Patienten waren älter, häufiger männlich und Raucher als Patienten ohne diese Lungenerkrankung. Die kardiovaskuläre Mortalität der COPD-Patienten war um den Faktor 1,54 höher.

Im Übrigen spielte es für die Wirksamkeit der Antikoagulanzien keine Rolle, ob der Patient COPD hatte oder nicht: Apixaban war auch bei COPD-Patienten wirksamer und sicherer als Warfarin.

Quelle: Kongress der American Thoracic Society (ATS) vom 15-20. Mai in Denver

\section{„Ältere" anders behandeln?}

Mit jeden zusätzlichen fünf Jahren Lebensalter sei der Analyse zufolge die Wahrscheinlichkeit, nicht mehr auf die Asthmabehandlung anzusprechen, um 13 Prozent gestiegen, alle zehn Jahre um 27 Prozent. Die statistisch signifikanten Unterschiede blieben auch dann bestehen, wenn als Vergleichsaltersgrenze statt 30 Jahre ein Alter von 38 Jahren gewählt wurde.

Darüber hinaus waren weitere Parameter mit einem Versagen der Therapie alterskorrigiert assoziiert. Dazu gehörten ein verringerter exspiratorischer Spitzenfluss (PEF) und ein erniedrigter Wert für die Einsekundenkapazität $\left(\mathrm{FEV}_{1}\right)$ sowie eine seit mehr als 15 Jahren bestehende Asthmaerkrankung. Zwar war ein Therapieversagen bei Frauen häufiger (15,2 vs. $11,7 \%)$, doch war dieser Unterschied nicht signifikant.

Einschränkend weisen die Ärzte darauf hin, dass es sich bei der Studiengruppe letztlich um ,jüngere Ältere" handelte, da nur etwa zehn Prozent aller Teilnehmer älter als 50 waren und nur sehr wenige über 65 .

In prospektiven Studien solle nun geprüft werden, ob ältere Asthmapatienten von einer Therapie profitierten, die sich von der bei jüngeren Patienten unterscheidet.

Peter Leiner

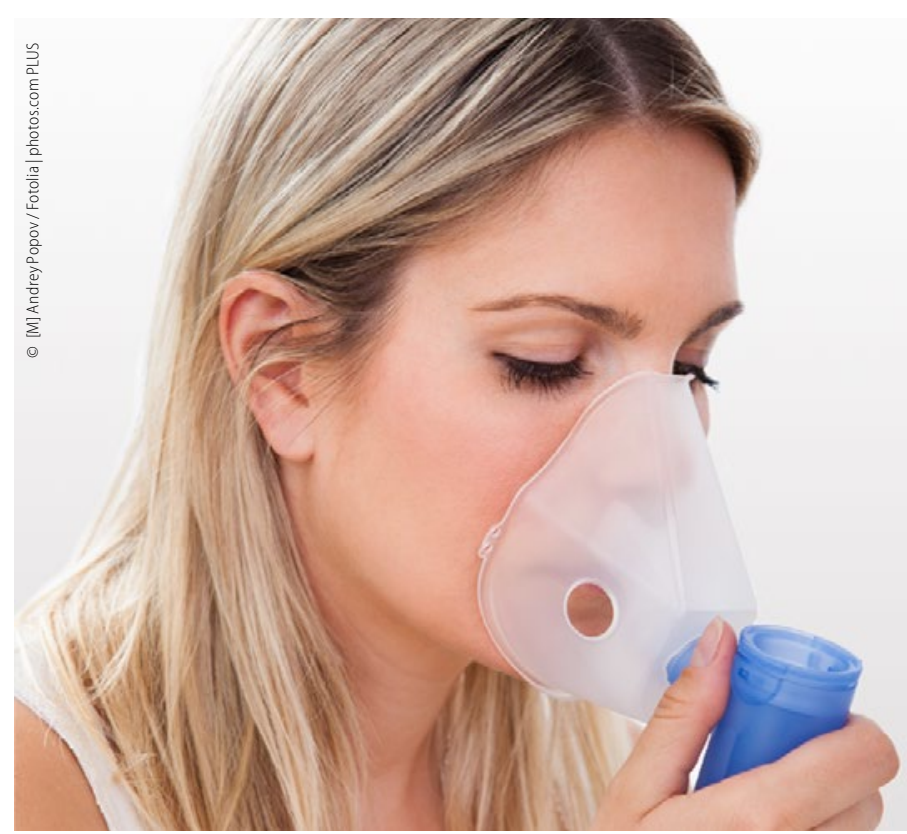

„Ältere“ Asthmapatienten entwickelten in dieser Studie trotz Therapie eher Exazerbationen und eine schlechtere Lungenfunktion.... 